\title{
Receptivity of a TVD Scheme in Incompressible Flow Analysis
}

\author{
By Byeong Rog SHIN \\ Institute of Fluid Science, Tohoku University, Sendai, Japan
}

(Received August 20th, 2002)

\begin{abstract}
A TVD upwind scheme originally designed for compressible flow is applied to the SMAC finite-difference method for incompressible flow analysis. The receptivity and validity of this application are investigated by an evaluation of the accuracy, stability and convergence rate for the SMAC method combined with the TVD scheme. Using this method, three-dimensional developing entry flows through a square-curved duct are calculated and compared with available experimental data as well as some computational results obtained by QUICKs and third-order upwind schemes. Such comparisons show that the numerical method applying the TVD scheme has the highest computational efficiency without a sharp loss of accuracy, resulting in confidence in the application this scheme to incompressible flow computations.
\end{abstract}

Key Words: TVD Scheme, QUICK Scheme, Incompressible Navier-Stokes Equation, Curved Duct Flow, Secondary Flow

\section{Introduction}

Recently, thanks to the rapid progress of computer capability and the development of reliable computational fluid dynamics codes, it has become possible to accurately analyze practical engineering flows. Many engineering flow fields, however, such as blade passages in turbomachinery or the flow fields around moving devices have geometrically complex computational domains. Furthermore, flows may be characterized by unsteadiness, turbulence and convectiondomination. In practical computations, therefore, supercomputing with a large number of grid points and iterations is required, and computational fluid dynamics codes with high efficiency, accuracy and numerical stability are required.

In the computation of convection-dominated flows, the application of central difference approximations to the discretization of convection terms leads to numerical instability and non-physical oscillations from time to time. To circumvent these instabilities, therefore, the upwind method for the convection term in Navier-Stokes equations is widely used. Among upwind methods, higher-order upwind schemes such as the QUICK family schemes ${ }^{1,2)}$ and the third-order upwind scheme are commonly used in calculations of incompressible flow because they guarantee the high accuracy of the solution despite the effect of numerical diffusion by upwinding. On the other hand, the total variation diminishing (TVD) schemes ${ }^{3,4)}$ are quite robust upwind schemes used for compressible flow problems. Actually, the application of TVD schemes to these problems has been very successful in the past decade. The primary reason for the increased popularity of high-speed flow simulation is that the TVD schemes utilize a limiting procedure to remove the local extremes, making this scheme capable of automatically generating nonoscillation and obtaining favorable approximations to shocks and contact discontinuities. Generally, however, this scheme yields a solution locally containing first-order accuracy due to the limiting procedure.

The purpose of this paper is to investigate the receptivity and validity for application of the TVD scheme to solving incompressible flow problems. The numerical method applied to the TVD scheme is an efficient implicit SMAC scheme, 5,6 ) which was developed by the author to solve incompressible Navier-Stokes equations in curvilinear coordinates. Using this method, a simulation of laminar and turbulent entry flow through a three-dimensional (3-D) bent square-duct ${ }^{7,8}$ ) is performed because it is the most appropriate fluid dynamics device for understanding complex flow phenomena. This entry flow is often used as a benchmark problem to examine numerical schemes.

In this study, comparisons of predicted results from the TVD and other high-order upwind schemes with experimental data are provided to show the validity and feasibility of the present finite-difference method combined with the TVD scheme. It is also shown that even though the TVD scheme is designed for hyperbolic equations, its use is greatly advantageous in the simulation of practical incompressible flows where it is difficult to apply QUICK and MQUICK schemes as well as the third-order upwind scheme.

\section{Fundamental Equations and Numerical Method}

\subsection{Fundamental equations of curvilinear coordinates}

The fundamental equations are the continuity equation and incompressible Navier-Stokes equations of volume fluxes $J U_{\ell}$ in curvilinear coordinates. They can be written in conservative forms as ${ }^{6)}$ :

$$
D \equiv \frac{\partial}{\partial \xi_{i}}\left(J U_{i}\right)=0
$$

(c) 2003 The Japan Society for Aeronautical and Space Sciences 


$$
\frac{\partial}{\partial t}\left(J U_{\ell}\right)+L\left(J U_{\ell}, p\right)=0 \quad(\ell=1,2,3)
$$

where,

$$
\begin{aligned}
L\left(J U_{\ell}, p\right) \equiv & \frac{\partial}{\partial \xi_{i}}\left(J U_{i} U_{\ell}\right)-J U_{i} \boldsymbol{u} \cdot \frac{\partial}{\partial \xi_{i}} \nabla \xi_{\ell}+\tilde{g}_{\ell i} \frac{\partial p}{\partial \xi_{i}} \\
& +v \epsilon_{\ell i j} \frac{\partial}{\partial \xi_{i}} \tilde{h}_{j k} J Z_{k}
\end{aligned}
$$

$\tilde{g}_{i j}=J g_{i j}$ and $\tilde{h}_{i j}=h_{i j} / J . \boldsymbol{u}, p, v$ and $\epsilon_{\ell i j}$ are the velocity, pressure, kinematic viscosity and permutation tensor, respectively. The Jacobian $J$ and metrics $g_{i j}$ and $h_{i j}$ of the transformation from Cartesian coordinates $x_{i}$ to general curvilinear coordinates $\xi_{i}$ are $J=\partial(x, y, z) / \partial(\xi, \eta, \zeta)$, $g_{i j}=\nabla \xi_{i} \cdot \nabla \xi_{j}$ and $h_{i j}=\partial x_{k} / \partial \xi_{i} \cdot \partial x_{k} / \partial \xi_{j}$, respectively. Relations between the physical velocity $u_{i}$ in $x_{i}$ space and the contravariant velocity $U_{i}$ in $\xi_{i}$ space are $U_{i}=\left(\partial \xi_{i} / \partial x_{j}\right) u_{j}$ and $u_{i}=\left(\partial x_{i} / \partial \xi_{j}\right) U_{j}$ using summation convention. Similarly, the contravariant vorticity $Z_{i}$ and physical vorticity $\zeta_{i}$ are defined as $Z_{i}=\left(\partial \xi_{i} / \partial x_{j}\right) \zeta_{j}$ and $\zeta_{i}=\nabla \times \boldsymbol{u}=\left(\partial x_{i} / \partial \xi_{j}\right) Z_{j}$. In Eq. (2), the momentum equations of volume fluxes $J U_{\ell}$ correspond to the equations of flows through each cell side. Therefore, for the usual body fitted curvilinear coordinate grid, the boundary condition can be imposed very easily.

In the turbulent flow computation, the LES (large-eddy simulation) method for subgrid-scale (SGS) turbulence is applied by adding the SGS stress term of $-J \nabla \xi_{\ell}\left(\nabla \cdot \overline{\boldsymbol{u}^{\prime} \boldsymbol{u}^{\prime}}\right)$ to the right-hand side of Eq. (3). In the present study, Smagorinsky's SGS turbulence model ${ }^{9)}$ employing the eddy viscosity $v_{s}$ is used as:

$$
-\overline{u_{i}^{\prime} u_{j}^{\prime}}=v_{s}\left(\frac{\partial u_{i}}{\partial x_{j}}+\frac{\partial u_{j}}{\partial x_{i}}\right)-\frac{2}{3} \delta_{i j} k_{s}
$$

$$
v_{s}=\left(C_{s} \Delta\right)^{2} \sqrt{2 \bar{S}_{i j} \bar{S}_{i j}}, \quad \bar{S}_{i j}=\frac{1}{2}\left(\frac{\partial u_{i}}{\partial x_{j}}+\frac{\partial u_{j}}{\partial x_{i}}\right)
$$

$\Delta$ is the grid size, and $C_{s}$ is the SGS coefficient. In the actual LES analysis, the turbulent energy $k_{s}$ is ignored because it is so small.

Applying the fractional step method to Eq. (2), we have the following explicit SMAC scheme of curvilinear coordinates:

$$
\begin{gathered}
J U_{\ell}^{*}=J U_{\ell}^{n}-\Delta t L\left(J U_{\ell}, p\right)^{n} \\
J U_{\ell}^{n+1}=J U_{\ell}^{*}-\Delta t \tilde{g}_{\ell i} \frac{\partial \phi}{\partial \xi_{i}}
\end{gathered}
$$

Then, taking the divergence of Eq. (7) and using Eq. (1) for $J U_{\ell}^{n+1}$, we obtain the elliptic equation of pressure increment $\phi$ :

$$
\begin{aligned}
\frac{\partial}{\partial \xi_{\ell}}\left(\tilde{g}_{\ell i} \frac{\partial \phi}{\partial \xi_{i}}\right) & =\frac{1}{\Delta t} \frac{\partial}{\partial \xi_{\ell}}\left(J U_{\ell}^{*}\right) \\
p^{n+1} & =p^{n}+\phi
\end{aligned}
$$

where, the asterisk $*$ denotes the intermediate time level and $n$ denotes the quantities at time $t=n \Delta t$.

In this scheme, the dependent variables $J U_{\ell}$ and $p$ are defined at different points of a staggered square grid in $\xi$ space. Using such a staggered grid is advantageous in that the occurrence of spurious errors is completely removed.

\subsection{Implicit SMAC scheme}

Now, Eq. (6) is extended to an implicit form by applying the delta-form approximate-factorization method ${ }^{10)}$ and partially including the viscous term in the left-hand side. Therefore, the momentum equations of the present implicit SMAC scheme are as follows, for instance, with regard to $J U$ in the $\xi$-direction:

where,

$$
\begin{aligned}
& {\left[1+\Delta t\left(\frac{\partial}{\partial \xi} U^{n}-v \frac{\partial}{\partial \xi} \tilde{h}_{22} \tilde{h}_{33} \frac{\partial}{\partial \xi}\right)\right] \cdot\left[1+\Delta t\left(\frac{\partial}{\partial \eta} V^{n}-v \frac{\partial}{\partial \eta} \tilde{h}_{33} \frac{\partial}{\partial \eta} \tilde{h}_{11}\right)\right]} \\
& {\left[1+\Delta t\left(\frac{\partial}{\partial \zeta} W^{n}-v \frac{\partial}{\partial \zeta} \tilde{h}_{22} \frac{\partial}{\partial \zeta} \tilde{h}_{11}\right)\right] \Delta J U^{*(m)}=R H S_{1}^{(m-1)}}
\end{aligned}
$$

where,

$$
\left.\begin{array}{l}
R H S_{1}^{(0)}=-\Delta t L(J U, p)^{n} \\
R H S_{1}^{(m)}=R H S_{1}^{(m-1)}+\Delta R H S_{1}^{(m)} \\
\Delta R H S_{1}^{(m)}=-\left(J U^{(m)}-J U^{(m-1)}\right)-\frac{1}{2} \Delta t\left(F_{1}^{(m)}-F_{1}^{(m-1)}\right) \quad \text { for } \quad m \geq 1
\end{array}\right\}
$$

and

$$
\begin{gathered}
F_{1}=\frac{\partial}{\partial \xi_{i}}\left(J U_{i} U\right)+\tilde{g}_{\ell i} \frac{\partial p}{\partial \xi_{i}}+v \epsilon_{\ell i j} \frac{\partial}{\partial \xi_{i}}\left(\tilde{h}_{j k} J Z_{k}\right) \\
J U^{*(m)}=J U^{(m-1)}+\Delta J U^{*(m)} \\
J Z_{\ell}=\epsilon_{\ell i j} \frac{\partial}{\partial \xi_{i}}\left(\tilde{h}_{j k} J U_{k}\right) \quad(\ell=1,2,3)
\end{gathered}
$$

When the solution converges, Eq. (10) is always reduced to the Crank-Nicholson method. In the same way, Eqs. (7), (8) and (9) can be written as follows:

$$
\begin{gathered}
J U_{\ell}^{(m)}=J U_{\ell}^{*(m)}-\frac{1}{2} \Delta t \tilde{g}_{\ell i} \frac{\partial \phi^{(m)}}{\partial \xi_{i}} \\
\frac{\partial}{\partial \xi_{\ell}}\left(\tilde{g}_{\ell i} \frac{\partial \phi^{(m)}}{\partial \xi_{i}}\right)=\frac{1}{\Delta t} \frac{\partial}{\partial \xi_{\ell}}\left(J U_{\ell}^{*(m)}\right)
\end{gathered}
$$




$$
p^{(m)}=p^{(m-1)}+\phi^{(m)}
$$

In Eqs. (10)-(14), the superscript $m$ denotes the number of Newton iterations. At $m=1, m-1=n$ and this scheme becomes an implicit SMAC scheme for steady-state flows. This delta-formed implicit SMAC scheme satisfies a diagonally dominant condition with the first-order upwind difference scheme and is TVD stable. This implicit SMAC scheme is also suitable for vector or vector parallel machines. ${ }^{11)}$

In the computation for unsteady flow, the solutions at each time step $J U_{\ell}^{n+1}$ and $p^{n+1}$ are obtained from Eqs. (10) to (14) of the Newton iteration. The right-hand sides of Eqs. (10) and (13) represent the residuals of Eqs. (2) and (1), respectively. $\Delta J U^{*(m)}$ and $\phi^{(m)}$ of the left-hand sides are the corrections of $J U$ and $p$ at each iteration. If the values of these residuals are close to zero, then the corrections also tend to zero and the solution converges. The accuracy of the solution depends only on the right-hand sides, and is independent of the left-hand sides.

Equation (10) can be solved by dividing it into three steps; each step is a problem which is solved by simultaneous linear equations with a tri-diagonal matrix by Gaussian elimination. Simultaneous linear Eq. (8) or (13) can be solved by the vectorized Tschebyscheff SLOR method, in which sweep directions are alternated as in the ADI method, which is suitable in supercomputing.

\section{QUICKs and TVD Schemes}

The second-order central-difference is basically used for space derivatives. However, for the convection term on the left-hand side of Eq. (10), the first-order upwind difference scheme is used to reduce computational effort and to accelerate convergency, while the higher-order upwind difference scheme is applied to the right-hand side to solve the high Reynolds number flow with accuracy and stability. Here, a simple one-dimensional equation is considered to explain the upwind schemes as

$$
\frac{\partial u}{\partial t}+\frac{\partial f(a, u)}{\partial x}=\frac{\partial u}{\partial t}+a \frac{\partial u}{\partial x}=0
$$

where, $a(u)=\partial f / \partial u$ is the propagating speed of waves or the transport speed of the convection term. The finitedifference equation of Eq. (15) can generally be written in the conservation form

$$
\begin{aligned}
& u_{i}^{n+1}+\lambda \theta\left(\hat{f}_{i+1 / 2}-\hat{f}_{i-1 / 2}\right)^{n+1} \\
& \quad=u_{i}^{n}-\lambda(1-\theta)\left(\hat{f}_{i+1 / 2}-\hat{f}_{i-1 / 2}\right)^{n}
\end{aligned}
$$

where, $\lambda=\Delta t / \Delta x, 0 \leq \theta \leq 1$, and $\hat{f}$ is the numerical flux function. In the case of the second-order centraldifference scheme, the numerical flux function is taken as $\hat{f}_{i+1 / 2}=a_{i+1 / 2}\left(u_{i}+u_{i+1}\right) / 2$.

\subsection{MQUICK and third-order upwind schemes}

As a general form of the upwind finite-difference scheme with fourth-order artificial dissipation, the numerical flux in Eq. (16) is written as:

$$
\hat{f}_{i+1 / 2}=a_{i+1 / 2}\left\{-(1+\alpha) u_{i-1}+\left(c_{1}+3 \alpha\right) u_{i}+\left(c_{1}-3 \alpha\right) u_{i+1}-(1-\alpha) u_{i+2}\right\} / c_{2}
$$

with $\alpha=\alpha_{0} \operatorname{sign}\left(a_{i+1 / 2}\right)$. In the QUICK scheme, ${ }^{1)}$ the values $\alpha_{0}=1, c_{1}=9$ and $c_{2}=16$ in Eq. (17) are chosen. Also, in the MQUICK scheme, ${ }^{2}$ which has better numerical stability and a higher convergence rate than the original QUICK scheme, $\alpha_{0}=4$ is generally adopted. On the other hand, for the third-order upwind scheme, $\alpha_{0}=1, c_{1}=7$ and $c_{2}=12$ are employed.

\subsection{TVD upwind scheme}

The higher-order TVD scheme is expressed as a sum of the first-order upwind scheme and correction terms which function to realize higher-order schemes, and then the correction terms are modified locally by a limiter in order to stabilize the solution. The numerical flux of the Chakravarthy-Osher TVD upwind scheme ${ }^{4)}$ becomes:

$$
\begin{aligned}
\hat{f}_{i+1 / 2}= & \left.\frac{f_{i}+f_{i+1}}{2}-\frac{D f_{i+1 / 2}^{+}-D f_{i+1 / 2}^{-}}{2}\right\} \text { 1st upwind } \\
& +\frac{1-\kappa}{4} \mathrm{~d} \tilde{\tilde{f}}_{i-1 / 2}^{+}+\frac{1+\kappa}{4} \mathrm{~d} \tilde{f}_{i+1 / 2}^{+} \quad\left(a_{i+1 / 2} \geq 0\right) \\
& -\underbrace{\frac{1-\kappa}{4} \mathrm{~d} \tilde{f}_{i+3 / 2}^{-}}_{\text {2nd upwind }}-\underbrace{\frac{1+\kappa}{4} \mathrm{~d} \tilde{\tilde{f}}_{i+1 / 2}^{-}}_{\text {2nd central }} \quad\left(a_{i+1 / 2}<0\right)
\end{aligned}
$$

Here, the flux-limited values of $\mathrm{d} f$ and the minmod function are determined by

$$
\mathrm{d} \tilde{\tilde{f}}_{i+1 / 2}^{ \pm}=\operatorname{minmod}\left(D f_{i+1 / 2}^{ \pm}, \quad b D f_{i+3 / 2}^{ \pm}\right)
$$

$$
\mathrm{d} \tilde{f}_{i+1 / 2}^{ \pm}=\operatorname{minmod}\left(D f_{i+1 / 2}^{ \pm}, b D f_{i-1 / 2}^{ \pm}\right)
$$

$\operatorname{minmod}(x, y)=\operatorname{sign}(x) \max [0, \min \{|x|, y \operatorname{sign}(x)\}]$ (19) and, $D f_{i+1 / 2}^{ \pm}=a_{i+1 / 2}^{ \pm} \Delta u_{i+1 / 2}, a^{ \pm}=(a \pm|a|) / 2, \Delta u_{i+1 / 2}=$ $u_{i+1}-u_{i}$. The parameter $\kappa$ is a linear combination parameter 


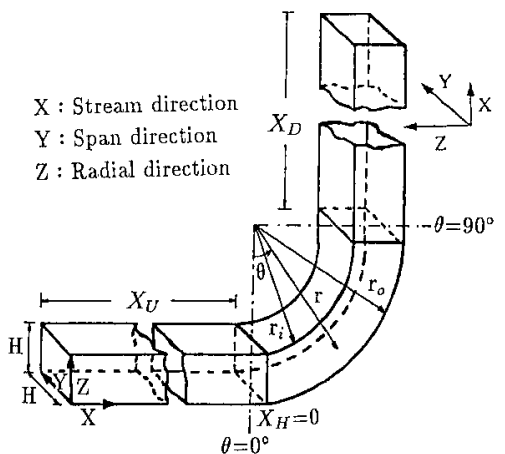

Fig. 1. Computational geometry of 90-degree bent duct.

determined by the range of $-1 \leq \kappa \leq 1$, and affects the accuracy of Eq. (18). According to the Taylor expansion, the truncation error for the unlimited forms of the TVD scheme in Eq. (18) is expressed as $-\frac{1}{12}(3 \kappa-1) \Delta x^{2} f_{x x x}+O\left(\Delta x^{3}\right)$. Therefore, we know that the TVD scheme has a third-order accuracy at $\kappa=1 / 3$, and that it becomes a second-order scheme at the other values of $\kappa$. Particularly for the $\kappa$ of $-1,0,1 / 2$ and 1, Eq. (18) becomes a second-order upwind scheme, Fromm's, ${ }^{12)}$ QUICK and central difference scheme, respectively. On the other hand, the slope of the flux in the minmod function of Eq. (19) is controlled by the limiter $b$. The range of $b, 1 \leq b \leq(3-\kappa) /(1-\kappa)$, is determined by the condition of TVD stability; as $b$ is larger, the region where the limiter function works becomes narrower.

\section{Numerical Results}

The numerical example considered in this study is a laminar, turbulent entry flow through a 3-D square-duct with a 90-degree bend as shown in Fig. 1. This bent duct flow is often used as a benchmark problem for 3-D flows, ${ }^{13,14)}$ and the curved square-duct is a basic element for actual flow devices. The computational geometry of the curved area is the same as the configuration studied experimentally by Taylor et al. ${ }^{7)}$ The length of $X_{U}$ and $X_{D}$ in Fig. 1 are $9 H$ and $5 H$, respectively. A no-slip boundary condition together with the Neumann condition for pressure was implemented on the solid wall boundary. The uniform flow condition at the inlet boundary was also prescribed. The control parameter $b$ of 4 and $\kappa$ of $1 / 3$ in Eq. (18) were used to guarantee the highest possible accuracy in the application of the TVD scheme.

First of all, in order to investigate the numerical stability and convergence rate for the TVD application in an incompressible flow computation, Fig. 2 presents iteration histories of the norm of divergence and pressure residual. The norm of divergence, Eq. (1), and pressure residual, Eq. (9), are defined as

$$
\text { Norm of Divergence }=\left(\frac{1}{N} \sum_{i j k} D^{2}\right)^{1 / 2}
$$

and

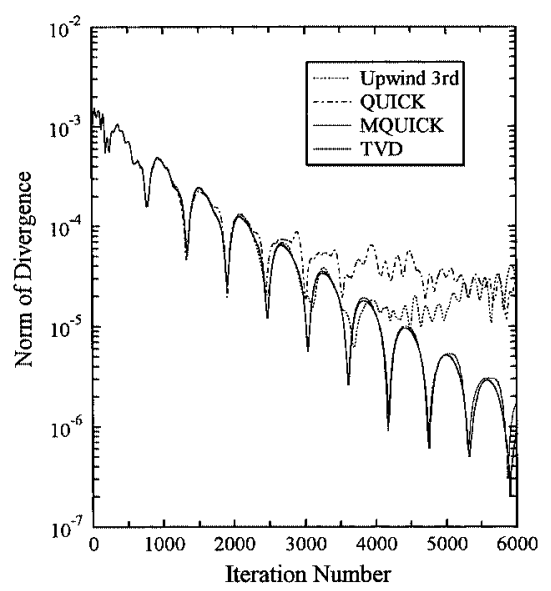

(a)

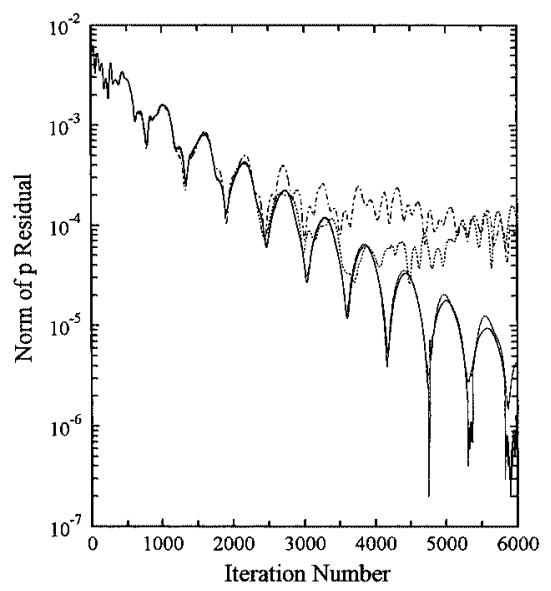

(b)

Fig. 2. Comparison of iteration history of (a) norm of divergence and (b) pressure residual.

$$
\text { Norm of } p \text { Residual }=\left(\frac{1}{N} \sum_{i j k} \phi^{2}\right)^{1 / 2}
$$

where, $N$ is the total number of grid points. $i, j$ and $k$ represent the index of each direction of the $\xi, \eta$ and $\zeta$, respectively. At the beginning of computation, the TVD scheme allowed an impulsive start with only the above boundary conditions. However, the other schemes required a starting value or careful attention to such factors as starting with a very small time increment and a prescription of velocity and pressure fields even though it is a laminar flow of $R e=790$, in which the Reynolds number $(R e)$ is based on the inlet mean velocity $\left(U_{0}\right)$ and entrance width $(H)$. Therefore, all computations to compare the convergence rates were performed with a starting value obtained from precomputation by the TVD scheme with 400 iterations.

The effect of applying the TVD scheme to the laminar flow with the above Reynolds number is remarkable in the iteration histories, where a body-fitted coordinate grid with $107 \times 41 \times 41$ points was used and streamwise spacing at the bend area was $2.5^{\circ}$. In the case of the third-order upwind and QUICK scheme, the norms are still in the range of the 

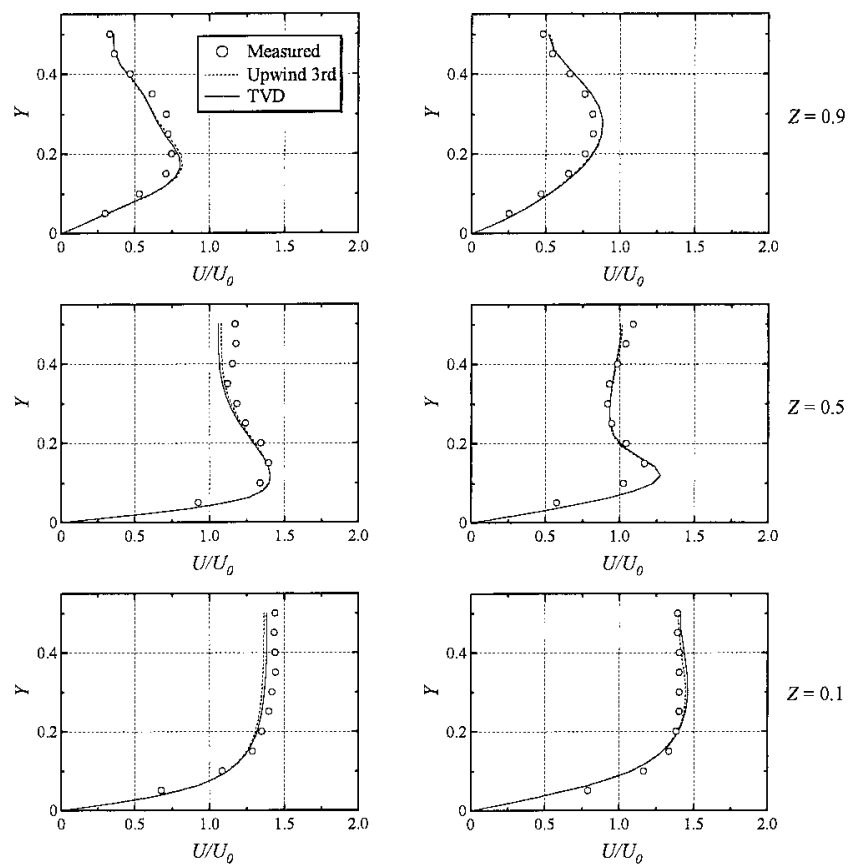

(a) $\theta=60^{\circ}$

(b) $X_{H}=2.5$

Fig. 3. Comparison of streamwise velocity profiles.
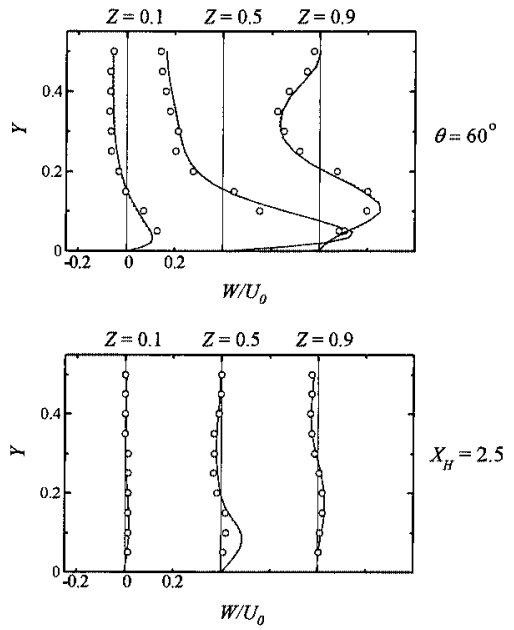

Fig. 4. Comparison of radial velocity profiles: - - - 3rd upwind; TVD scheme; o, measurements.

order of $10^{-5}$ after 6000 iterations and they are somewhat unstable. With the TVD scheme, however, a rapid convergence rate is obtained. In this laminar flow, the tendency of the stability and that of the convergence rate of the TVD scheme are similar to those of the MQUICK scheme, which is an improved QUICK scheme for incompressible flow.

Figures 3 and 4 show some comparisons of streamwise velocity $(U)$ and radial velocity $(W)$ profiles along the spanwise direction $Y$ with experiments ${ }^{7)}$ at several streamwise locations. The agreement between predictions and measurements is quite good at most locations. The present computations capture the qualitative features of the flow well. The discrepancy between the result obtained by the TVD scheme and that by the third-order upwind scheme is very small; thus

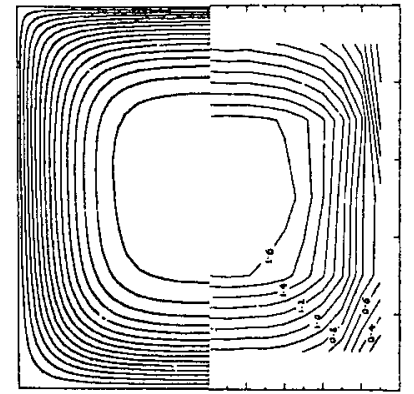

$X_{H}=-0.25$

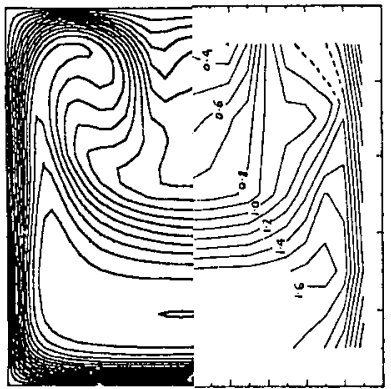

$\theta=77.5^{\circ}$
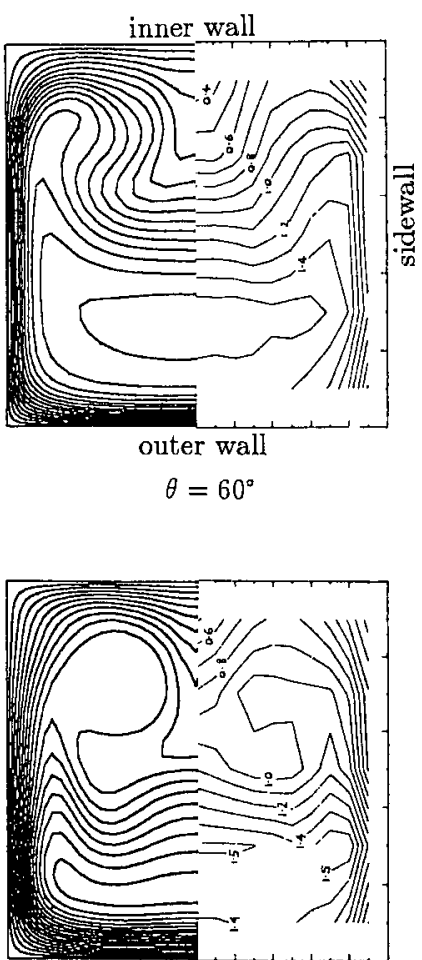

$X_{H}=2.5$
Fig. 5. Comparison of streamwise velocity contours at several streamwise stations.

Left: computation, Right: experiments.

showing that, from Figs. 2 through 4, the numerical method using the TVD scheme is more efficient than the others.

Figure 5 shows computed streamwise velocity contours compared with contours derived from the measurements ${ }^{7)}$ at some streamwise locations. The results of the TVD scheme show good quantitative agreement with measurements as a whole. It shows that the center of the high-velocity flow moves to the outer wall with the angle of $\theta$ and up to about $2 \mathrm{H}$ downstream of the bend, which is due to the secondary flow formed by the curvature. At 60 and $77.5^{\circ}$ in the bend, the computed velocities gradually roll up the flow into a passage vortex. Then, at $X_{H}=2.5 H$ downstream from the bend, they show the decay of the vortex. These redistributions of the streamwise velocity agree very well with experiments.

Figures 6(a) and (b) show the distribution of the order of accuracy of applying the TVD scheme to a laminar flow. These indicate that the accuracy is in the range over the second-order in all computational regions. Therefore, we know that there is no problem with regard to the sharp loss of accuracy, which will occur due to the working of the minmod function as we experienced in the compressible flow computation.

In a manner similar to the laminar flow calculation, turbulent flow with a Reynolds number of 40,000 was computed. A body-fitted coordinate grid with $135 \times 61 \times 61$ points was used, and the streamwise spacing at the bend area was $2^{\circ}$. In the present turbulent flow simulation, QUICK and even 


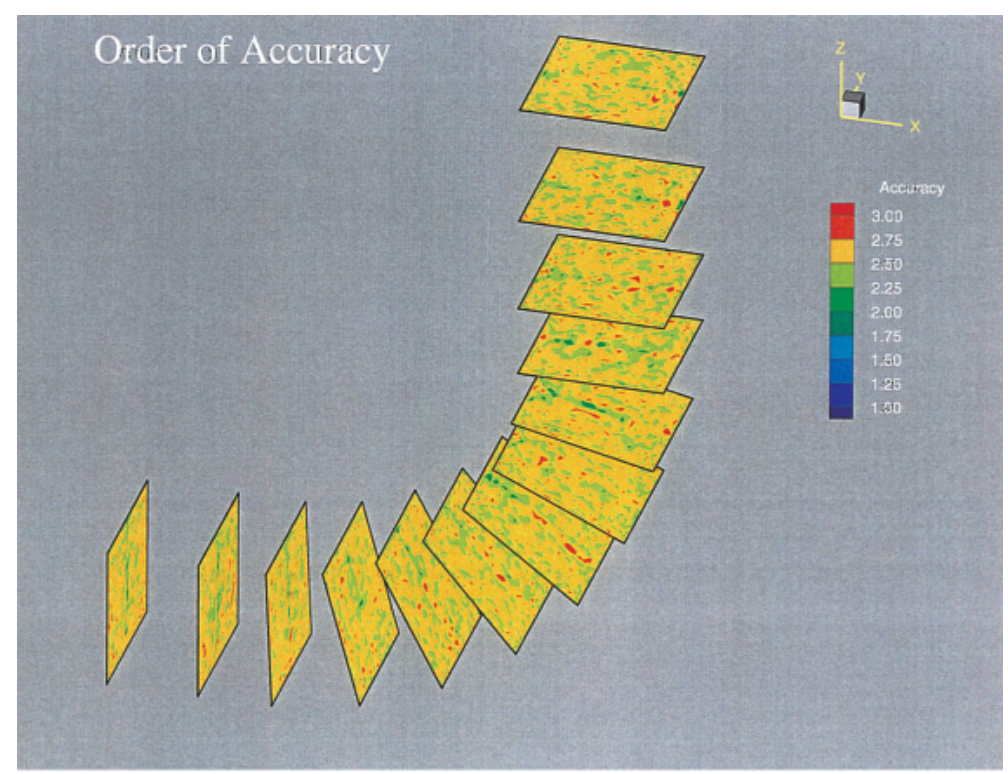

(a)

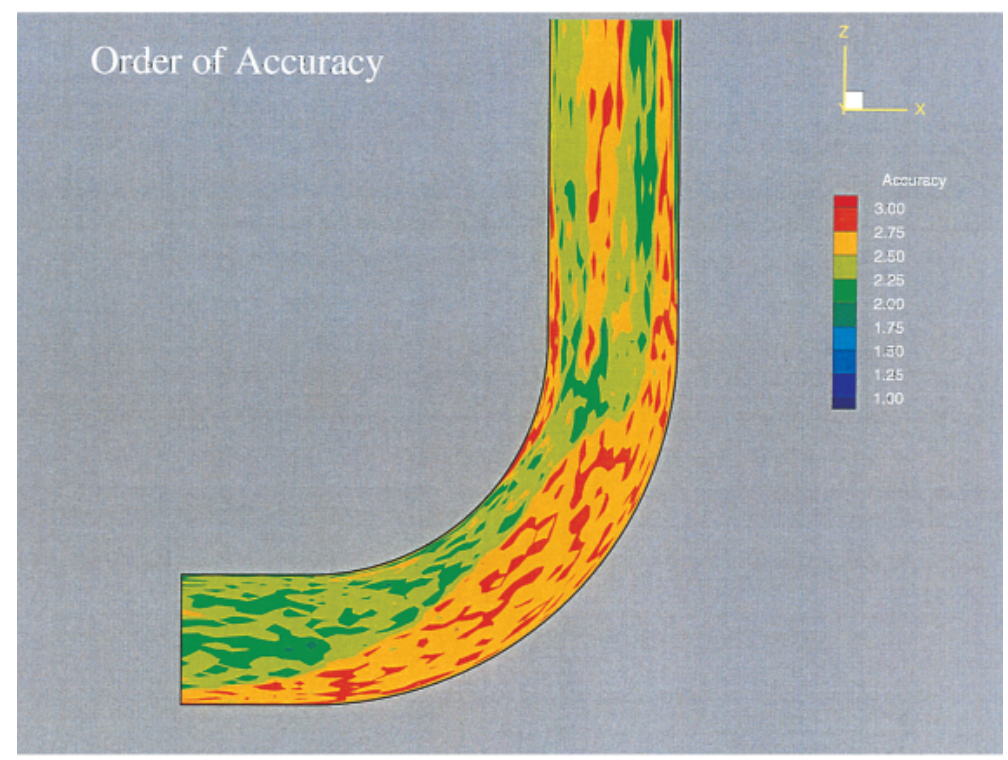

(b)

Fig. 6. Distribution of the order of accuracy for TVD scheme. (a) several streamwise cross sections, (b) symmetry plane.

MQUICK upwind schemes could not reduce the numerical instability at this Reynolds number and strong curved flow field although they well simulated the above laminar flows. This is because, even though the incompressible flow is milder than the compressible flow, an unsteady turbulent flow with a high Reynolds number sometimes leads to the local extreme on the account of geometrical effects such as squared corners and strong curvature.

The steady flow computation was first carried out to obtain the starting value for unsteady computation. Then unsteady turbulent flow computation was performed with the CrankNicholson method and LES method for SGS turbulence. The Smagorinsky constant $C_{s}$ of 0.1 is chosen in Eq. (5), and the grid scale $\Delta$ is modified to $\Delta=\min \left(\Delta_{\xi}, \Delta_{\eta}, \Delta_{\zeta}\right)$ be- cause the diagonal volume length or geometric mean length results in overestimation of the SGS viscosity for the present relatively coarse grid which has the above-mentioned grid points and a grid size of $0.005 H$ as the smallest value in both the $Y$ and $Z$-directions. Additionally, to take near-wall effects into account, the Van Drist wall-damping function $f_{d}$ $\left(=1-\exp \left(-y^{+} / A^{+}\right)\right)$was imposed on $v_{s}$ in Eq. (5) with the empirical coefficient $A^{+}$of 26 .

Figures 7 and 8 show some comparisons of time-averaged streamwise velocity and secondary velocity profiles along the $Y$ direction with LDV measurements ${ }^{7)}$ at several streamwise and radial locations. As seen in this figure, comparisons of computed streamwise velocities with measurements are quite good everywhere. In this turbulent flow, the strength of 

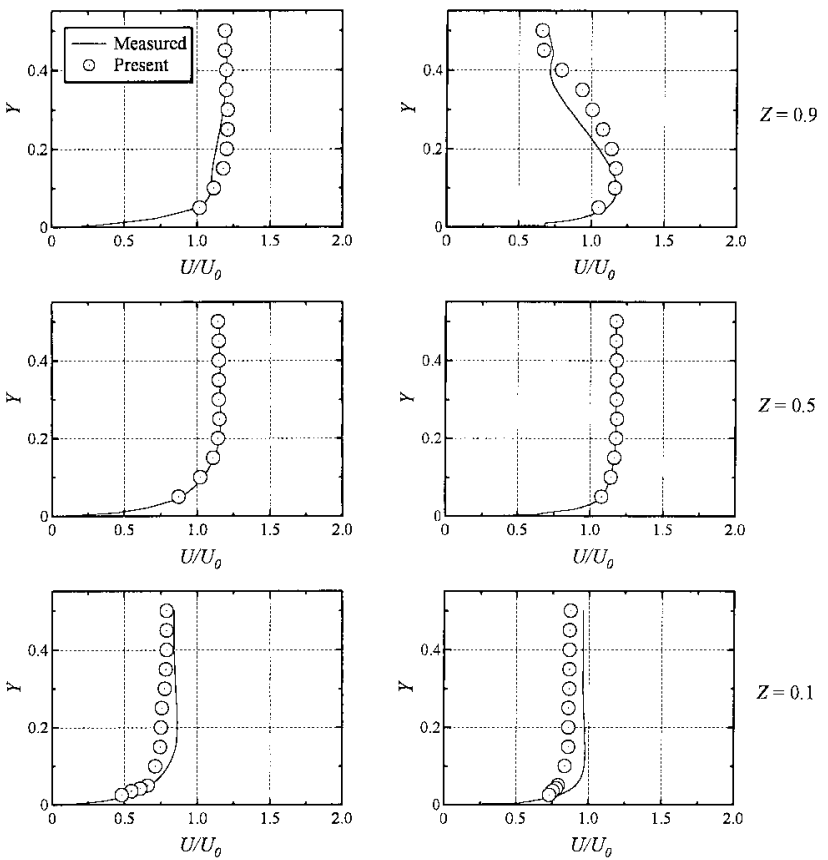

(a) $\theta=30^{\circ}$

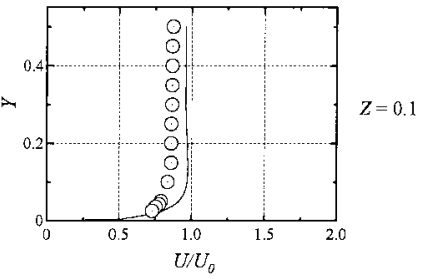

(b) $\theta=77.5^{\circ}$

Fig. 7. Comparison of time-averaged streamwise velocity profiles (turbulent flow).
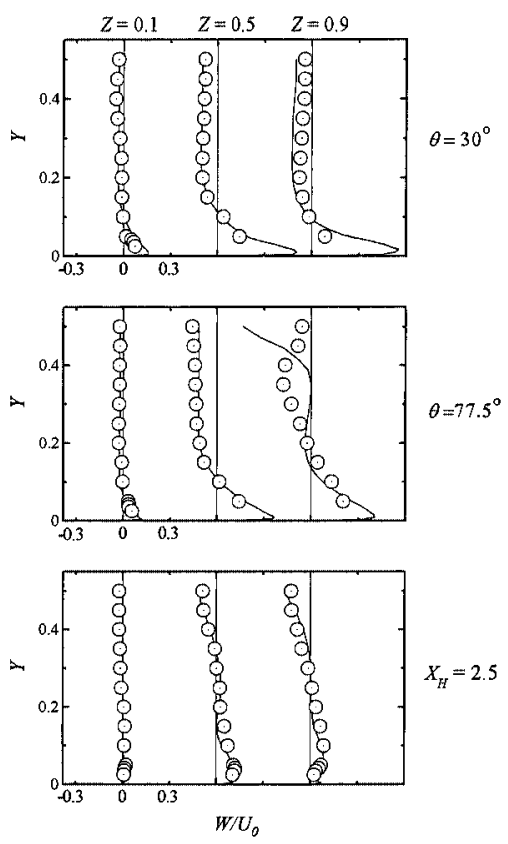

Fig. 8. Comparison of time-averaged radial velocity profiles (turbulent flow).

— , TVD scheme; o, measurements.

the secondary flow and the redistribution of the streamwise flow is smaller than that of the laminar flow in Figs. 3 and 4 due to the difference in the thickness of the inlet boundary layers.

Figure 9 presents the wall pressure distribution along the streamwise $\theta$-direction. Predicted pressure coefficients $C_{p}=2\left(p-p_{\text {ref }}\right) / \rho U_{0}^{2}$ with $p_{\text {ref }}$ of the pressure at $0^{\circ}, Z=0$
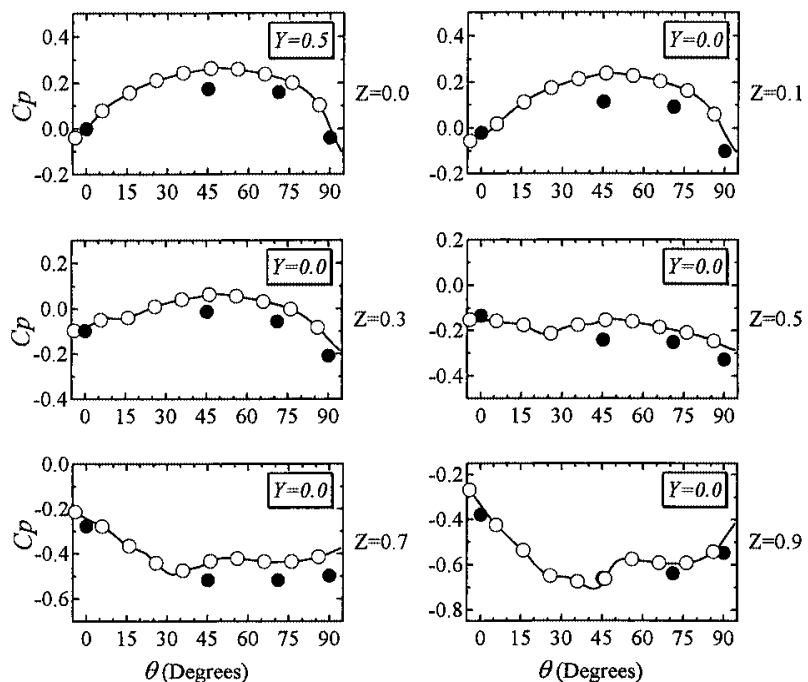

Fig. 9. Comparison of time-averaged wall pressure distributions (turbulent flow).

$\_$, TVD scheme; $\bullet$, measurements.

and the mid-span compare very well with measurements. An adverse pressure gradient develops on the outside wall (at $Z=0.1$ ) up to $\theta \approx 50^{\circ}$, and then the pressure gradient is reversed with $\theta$ due to flow deceleration and acceleration as shown in Fig. 7. On the inside wall, the pressure gradient shows a converse trend, as expected. Relatively high pressure is distributed near the outside wall and pressure loss recovers downstream of the bend.

Similarly to Fig. 5, Fig. 10 shows computed timeaveraged secondary velocity vectors, pressure contours, streamwise velocity contours and experimental results at several locations. At 30 and $77.5^{\circ}$ in the bend, development of the secondary flow results in a roll up into a vortex, and then this vortex gradually decays downstream. The present turbulent flow computation also captures the qualitative features of the flow well, as investigated so far.

\section{Conclusions}

The receptivity and validity for applying the TVD scheme to solving incompressible flow problems were investigated. The numerical method applied to the TVD scheme for this investigation is the efficient implicit SMAC scheme, previously developed to solve incompressible Navier-Stokes equations in general curvilinear coordinates.

Using the method, a 3-D developing entry laminar and turbulent flow through a square duct with a 90-degree bend was calculated and compared with numerical results obtained by QUICKs and the third-order upwind schemes and LDV measurements. Predictions by the TVD scheme showed good agreement with the experiments. Accurate simulations of 3D complex flow characteristics including the redistribution of the streamwise velocity and strong secondary motion in the bend were successfully shown.

In the comparison of stability and convergence rate with 

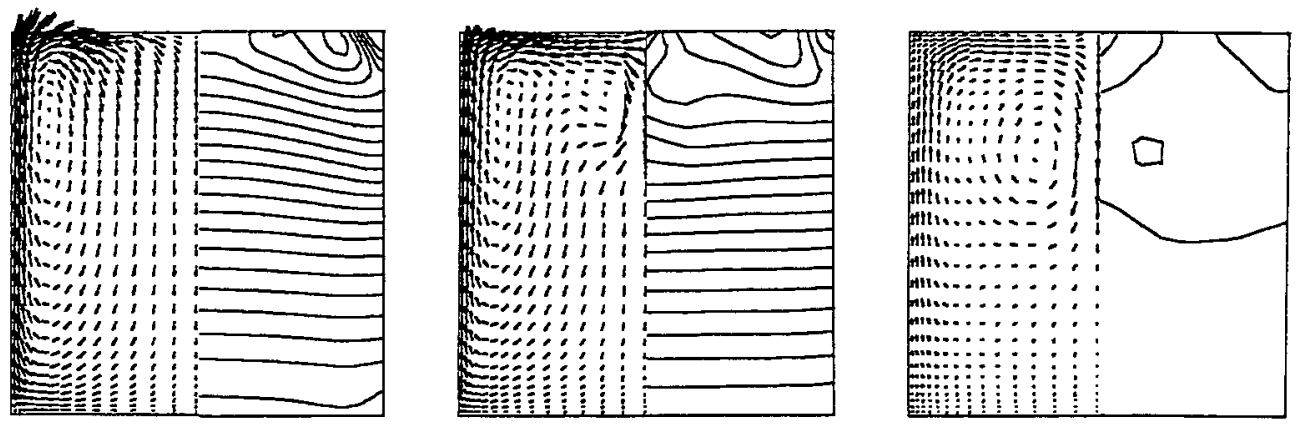

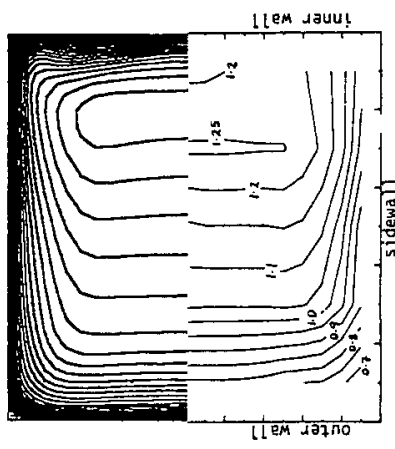

(a) $\theta=30^{\circ}$

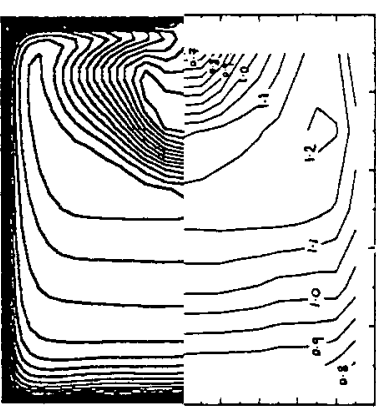

(b) $\theta=77.5^{\circ}$

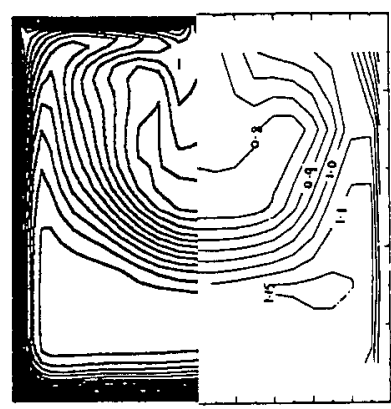

(c) $X_{H}=2.5$

Fig. 10. Turbulent flowfield at several streamwise stations (turbulent flow).

Upper left: secondary velocity vectors, upper right: pressure contours,

lower left: streamwise velocity contours, lower right: experiments.

QUICKs and the third-order upwind scheme, the implicit method applying the TVD scheme was found to be the most stable and to have the highest computational efficiency. It was confirmed that there is no sharp drop in accuracy due to the function of a limiting procedure in the TVD scheme. This limiting procedure, however, plays an important role in simulating practical incompressible flows with local extremes. In view of the results so far obtained, the TVD scheme can be used with confidence to solve incompressible flow problems.

\section{References}

1) Leonard, B. P.: A Stable and Accurate Convective Modeling Procedure Based on Quadratic Upstream Interpolation, Comput. Methods Appl. Mech. Eng., 19 (1979), pp. 59-98.

2) Shin, B. R., Ikohagi, T. and Daiguji, H.: A Modified QUICK Scheme with Good Stability and High Convergence Rate, Comput. Fluid Dyn. J., 7 (1998), pp. 283-299.

3) Harten, A.: High Resolution Schemes for Hyperbolic Conservation Laws, J. Comput. Phys., 49 (1983), pp. 357-393.

4) Chakravarthy, S. R. and Osher, S.: A New Class of High Accuracy TVD Schemes for Hyperbolic Conservation Laws, AIAA Paper 850363, 1985.

5) Shin, B. R., Ikohagi, T. and Daiguji, H.: An Unsteady Implicit SMAC Scheme for Two-Dimensional Incompressible Navier-Stokes Equations, JSME Int. J. Ser. B, 36 (1993), pp. 598-606.
6) Shin, B. R., Ikohagi, T. and Daiguji, H.: An Implicit Finite-Difference Scheme for the Incompressible Navier-Stokes Equations Using an Improved Factored Scheme, Comput. Fluid Dyn. J., 4 (1995), pp. 191208.

7) Taylor, A. M. K., Whitelaw, J. H. and Yianneskis, M.: Curved Ducts with Strong Secondary Motion: Velocity Measurements of Developing Laminar and Turbulent Flow, ASME J. Fluid Eng., 104 (1982), pp. 350-359.

8) Cabuk, H., Sung, C. H. and Modi, V.: Explicit Runge-Kutta Method for Three-Dimensional Internal Incompressible Flows, AIAA J., 30 (1992), pp. 2024-2031.

9) Smagorinsky, J.: General Circulation Experiments with the Primitive Equations, Mon. Weath. Rev., 91 (1963), pp. 99-164.

10) Beam, R. M. and Warming, R. F.: An Implicit Factored Scheme for the Compressible Navier-Stokes Equations, AIAA J., 16 (1978), pp. 393-402.

11) Shin, B. R., Ikohagi, T. and Daiguji, H.: An Implicit Finite Difference Scheme for the Incompressible Navier-Stokes Equations Using an Improved Factored Scheme, Comput. Fluid Dyn. J., 4 (1995), pp. 191-208.

12) Chakravarthy, S. R.: Development of Upwind Schemes for the Euler Equations, NASA CR 4043, 1987.

13) Govindan, T. R., Briley, W. R. and McDonald, H.: General ThreeDimensional Viscous Primary/Secondary Flow Analysis, AIAA J., 29 (1991), pp. 361-370.

14) Rosenfeld, M., Kwak, D. and Vinokur, M.: A Fractional Step Solution Method for the Unsteady Incompressible Navier-Stokes Equations in Generalized Coordinate Systems, J. Comput. Phys., 94 (1991), pp. 102-137. 